\title{
RESPUESTA DIFERENCIAL DE DOS ESPECIES DE EUCALIPTO A LA ACLIMATACIÓN POR K Y RIEGO EN VIVERO
}

\author{
Garau, Ana ${ }^{6}$; Guarnaschelli, Ana ${ }^{6}$ y Lemcoff, Jorge ${ }^{7}$
}

\section{RESUMEN}

El estrés hídrico es la principal restricción abiótica que afecta el establecimiento de los plantines forestales. Con el objetivo de evaluar el efecto del potasio y la restricción hídrica se estudió respuestas morfológicas y fisiológicas de plantines de Eucalyptus camaldulensis y E. globulus tanto en plantas de vivero como en condiciones controladas de plantación. Los tratamientos en vivero fueron una combinación de dos niveles de $\mathrm{K}$ ( $\mathrm{K} 0$ y $\mathrm{K} 1)$ y dos de disponibilidad hídrica (Control-v y Estrés-v). Luego, se trasplantaron en contenedores de $200 \mathrm{~L}$ y se sometieron a dos niveles de disponibilidad hídrica durante un mes (Control-p y Estrés-p). En ambas condiciones se evaluó la biomasa aérea y radical e índices de alocación relativa de fotosintatos a las hojas y a las raíces. Al final del período de plantación se calcularon tasas de crecimiento relativo. Al término de la etapa de vivero y de plantación se realizaron curvas presiónvolumen y se evaluó el potencial osmótico a saturación y el módulo máximo de elasticidad de la pared celular. Para evaluar el grado de aclimatación de calculó un índice de plasticidad.

Al finalizar la etapa de vivero no se observó efecto significativo del $\mathrm{K}$ en ambas especies. La restricción hídrica no afectó la biomasa ni la alocación relativa en los plantines de E. globulus, mientras que la disminuyó en E. camaldulensis, aunque produjo una mayor alocación relativa hacia raíces. Ante la restricción hídrica ambas especies realizaron ajuste osmótico de similar magnitud pero en E. camaldulensis hubo, además, un ajuste elástico con endurecimiento de la pared celular. Al finalizar la etapa de plantación solo se observó modificaciones positivas en la biomasa, en la alocación relativa y en las tasas de crecimiento en las plantas de E. globulus del nivel Estrés-p. Por su parte, en $E$. camaldulensis las plantas $\mathrm{K} 1$ mostraron mayores tasas de crecimiento relativo en biomasa aérea y radical y una mayor alocación hacia hojas; las plantas Estrés-v mostraron mayores tasas de crecimiento en biomasa aérea, aumentando la alocación relativa a hoja.

Ante la restricción hídrica en plantación, no hubo modificaciones fisiológicas en E. globulus. En las plantas de E. camaldulensis se observó un ligero ajuste osmótico en las plantas K1 y un endurecimiento de la pared celular con la restricción hídrica en plantación. La mayor dosis de $\mathrm{K}$ (K1) y la restricción hídrica durante la etapa de vivero (Estrés-V) produjo modificaciones plásticas de distinto nivel, tanto fisiológicas como morfológicas, en los plantines de ambas especies de eucalipto. La disponibilidad hídrica presentó los mayores valores en el índice de plasticidad en todas las variables evaluadas en la etapa de vivero. En la etapa post-plantación, si bien el principal efecto es de la restricción hídrica aplicada en esa etapa, se observó diferencias entre las especies de eucalipto ya que predomina el efecto del nivel de $\mathrm{K}$ sobre el efecto del nivel hídrico en $E$. camaldulensis. Plantines de eucalipto con mayores niveles de $\mathrm{K}$ y restricción en la disponibilidad hídrica durante la etapa de vivero produjeron plantas aclimatadas morfológica y fisiológicamente que mostraron (en el caso de E. camaldulensis) mayores tasas de crecimiento en condiciones de restricción hídrica en plantación. Sin embargo, y considerando que en el caso de E. globulus las respuestas fueron distintas, e inclusive sin diferencias significativas con los controles, la aplicación de dichas prácticas culturales debería ajustarse en función de cada especie.

Palabras clave: Aclimatación, Vivero, Eucaliptos

\footnotetext{
${ }_{7}^{6}$ Cátedra de Dasonomía-Depto.Producción Vegetal, Facultad Agronomía.UBA-Argentina agarau@agro.uba.ar

${ }^{7}$ Inst. Soil, Water \& Environmental Sciences. Volcani Center, Israel
} 


\section{SUMMARY}

Water stress is a major abiotic constraint affecting the establishment of forest seedlings. In order to evaluate the effect of potassium and water restriction morphological and physiological responses of Eucalyptus camaldulensis and E. globulus both as seedlings and planted in controlled conditions were studied. Nursery treatments were a combination of two levels of $K(K 0$ and $K 1)$ and two water availability conditions (Control-v and Stress-v). Then, plants were transplanted into containers of $200 \mathrm{~L}$ and subjected to two levels of water availability for a month (Control-p and Stress-p). In both conditions shoot and root biomass and relative rates of photosynthate allocation to leaves and roots were evaluated. At the end of planting period relative growth rates were calculated. After the nursery stage and planting period pressure-volume curves were performed and osmotic potential at full turgor and the maximum modulus of elasticity of the cell wall were evaluated. To assess the degree of acclimation a plasticity index was calculated.

At the end of the nursery stage no significant effect of $\mathrm{K}$ was observed in both species. The water restriction did not affect biomass or relative allocation in seedlings of E. globulus, while decreased it in E. camaldulensis, although producing a greater relative allocation to roots. With water restriction both species performed an osmotic adjustment of similar magnitude, but in $E$. camaldulensis there was also an elastic adjustment of the cell walls.

After the planting period only positive changes in biomass, in the allocation and relative growth rates were observed in E. globulus E-p plants. Meanwhile, E. camaldulensis K1 plants showed higher relative growth rates of shoot and root biomass and increased allocation to leaves; Stress-v plants showed higher growth rates in biomass, increasing the relative allocation to leaves.

No physiological changes related to water restrictions were observed in E. globulus. In K1 plants of $E$. camaldulensis there was a slight osmotic adjustment and a hardening of the cell wall related to water restriction during plantation. The higher dose of $K(K 1)$ and the water restriction during the nursery stage (Stress-V) produced plastic changes (both physiological and morphological), in the seedlings of both eucalypts species. Water availability showed the highest plasticity index values in all variables during the nursery stage. In the post-planting period, although the main observed effects was related to the water restriction applied at that stage, there were differences between eucalypts species as a predominant effect of $\mathrm{K}$ level was observed in $E$. camaldulensis plants. Higher levels of $\mathrm{K}$ and restriction in the availability of water during the nursery stage produced Eucalyptus plants morphological and physiologically acclimated that showed (in the case of $E$. camaldulensis) higher growth rates under conditions of water restriction during the planting period. However, as in E. globulus responses were different, and even without significant differences with the Controls, the application of such cultural practices should be adjusted according to each species.

Keywords: Nursery, Eucalypts, Acclimation 


\section{INTRODUCCION}

Las plantaciones de Eucalyptus representan cerca del $25 \%$ de la superficie de plantaciones comerciales en Argentina (Garau, 2012). En la provincia de Buenos Aires Eucalyptus camaldulensis y $E$. globulus son dos de las principales especies utilizadas en forestaciones comerciales (Cozzo, 1995). Debido a su baja tolerancia al frío, las principales especies se plantan en la primavera, cuando la ocurrencia de situaciones de deficiencia hídrica durante el establecimiento pueden ser comunes (Soriano, 1991). La disponibilidad de agua es uno de los principales factores que limita el establecimiento y crecimiento de los plantines forestales. En condiciones de sequía las especies de Eucalyptus exhiben diferentes cambios morfológicos y fisiológicos que aumentan su tolerancia al déficit hídrico (Lemcoff et al., 1994; Sasse y Sands, 1996; Lemcoff et al., 2002; Guarnaschelli et al., 2003)

Las características morfológicas y fisiológicas de los plantines varían significativamente con las prácticas de producción en la etapa de vivero y con las condiciones ambientales (Burdett, 1990). La aclimatación, respuesta fenotípica modificable por el nivel de disponibilidad de recursos, es una técnica usada comúnmente en los viveros. La aclimatación por riego, por ejemplo, consiste en restringir la disponibilidad de agua en las etapas finales de ese período (Landis, 1989). De esta manera, plantas aclimatadas mostraron mejor comportamiento en condiciones de sequía en distintas especies de Eucalyptus (Reis et al., 1988; Sasse y Sands, 1996; Osorio et al., 1998; Guarnaschelli et al., 2003, 2006). Guarnaschelli et al. (2006) hallaron que plantas de Eucalyptus globulus de 5 meses sometidas a aclimatación con sequía en vivero mostraron mejor estado hídrico, mayor conductancia estomática y mayor supervivencia que plantas no aclimatadas.

Por su parte, la fertilización potásica durante el período de aclimatación en vivero resulta, no solo importante para la nutrición de la planta, sino que cumple también un rol relevante relacionado a la tolerancia al estrés hídrico. El potasio (K), está involucrado en la osmoregulación y en el cierre y apertura de estomas (Kozlowski y Pallardy, 1997). Al disminuir el potencial agua, la acumulación de solutos (Nielsen y Orcutt, 1996) puede permitir que las plantas mantengan un gradiente de potencial agua con un suelo que se va secando, y lograr una turgencia suficiente para mantener los estomas abiertos y posibilitar el intercambio de gases y el crecimiento (White et al., 2000). La capacidad de ajuste osmótico está influenciada por la disponibilidad de nutrientes. Plantines de Quercus prinus fertilizados con NPK mostraron mayor ajuste osmótico en condiciones de sequía (Kleiner et al., 1992). En diversos trabajos se ha observado que las plantas que recibieron dosis de $\mathrm{K}$ respondieron mejor a condiciones de estrés hídrico (van den Driessche 1992, Garau et al., 2004; Texeira et al., 2008; Guarnaschelli et al., 2010).

En Eucalyptus globulus ssp maidenii y en E.globulus ssp globulus, Plante et al. (2002) y Mema et al. (2003) observaron mayor crecimiento en biomasa aérea y radical en condiciones de restricción hídrica en plantines que recibieron una mayor dosis de $\mathrm{K}$ en el vivero. Por su parte, van den Driessche (1991) halló incremento en el número de nuevas raíces en plantines de Pseudotsuga menziesii sometidas a restricción hídrica, pero fertilizadas con potasio, mientras que Von Wernick y Lavado (2001) observaron menor daño por heladas en plantines de Eucalyptus grandis fertilizados con K. Chamshama y Hall (1987) establecieron que la fertilización con K incrementó el diámetro a nivel del cuello, el peso seco y el crecimiento del sistema radical en plantines de E. camaldulensis.

La producción de materia seca depende básicamente de la distribución de los carbohidratos en el área fotosintética y de la tasa de fijación de carbono por unidad de área foliar. La tasa de crecimiento relativa (TCR) es una medida inherente de la eficiencia de crecimiento y permite identificar si una planta logra su superioridad en crecimiento a través de mayor rendimiento fotosintético (mayor TAN), mayor distribución relativa de fotosintatos en el área foliar (mayor relación de área foliar), o por combinación de ambas (Lambers y Poorter, 1992). Es decir, el análisis de crecimiento aporta una manera de integrar las respuestas fisiológicas de plantas creciendo bajo diferentes condiciones medioambientales a través del tiempo. De esta manera provee de una poderosa herramienta para evaluar las primeras etapas del crecimiento de los plantines (Margolis y Brand, 1990).

En este trabajo se analiza el efecto de dos niveles de fertilización con $\mathrm{K}$ y dos niveles de 
riego aplicados en la etapa de vivero sobre respuestas morfológicas y fisiológicas de plantines de Eucalyptus camaldulensis y E. globulus. Los objetivos principales son evaluar como esos tratamientos modifican la morfología y crecimiento de los plantines, evaluar la performance de esas plantas después de la plantación con restricción de riego en un ensayo controlado y analizar si hay respuestas diferenciales a nivel de especie de eucalipto.

\section{MATERIALES Y METODOS}

El ensayo se realizó en el campo experimental de la Facultad de Agronomía de la Universidad de Buenos Aires (34 35' 27' LS, 58 29' 47" LW y 20 msnm). Se trabajó con Eucalyptus camaldulensis y Eucalyptus globulus, dos de las especies más plantadas en Argentina (Cozzo, 1995).

Se usó semilla de Eucalyptus camaldulensis procedencia Lake Coorong y de E. globulus procedencia Flinders Island provista por Kylisa Seeds Pty Ltd. (Australia).

Los plantines fueron producidos en contenedores de $660 \mathrm{~cm}^{3}$, con una mezcla de fibra de coco y compost de corteza de pino $(1: 1 \mathrm{v} / \mathrm{v})$ como sustrato. El experimento se realizó en un invernáculo abierto con una cubierta de polietileno transparente de $100-\mu \mathrm{m}$.

\section{Cuadro $\mathrm{N}^{\circ} 1$}

CARACTERIZACIÓN DE LAS PROCEDENCIAS DE SEMILLA EMPLEADAS

\begin{tabular}{|c|c|c|c|c|c|c|c|}
\hline \multirow[b]{2}{*}{ Especie } & \multirow[b]{2}{*}{ Origen } & \multirow[b]{2}{*}{ Latitud S } & \multirow[b]{2}{*}{ Longitud E } & \multirow[b]{2}{*}{$\begin{array}{l}\text { Altitud } \\
\text { (msnm) }\end{array}$} & \multirow{2}{*}{$\begin{array}{c}\text { Precipitación } \\
(\mathrm{mm})\end{array}$} & \multicolumn{2}{|c|}{ Temperatura } \\
\hline & & & & & & $\begin{array}{c}\text { Media Enero } \\
\left({ }^{\circ} \mathrm{C}\right)\end{array}$ & $\begin{array}{c}\text { Media Julio } \\
\left({ }^{\circ} \mathrm{C}\right)\end{array}$ \\
\hline E. globulus ssp. globulus & Flinders Island & $40^{\circ} 00^{\prime}$ & $148^{\circ} 07^{\prime}$ & 26 & 743 & 17,7 & 9,8 \\
\hline E. camaldulensis & Lake Coorong & $35^{\circ} 45^{\prime}$ & $142^{\circ} 23^{\prime}$ & 81 & 452 & 22,8 & 8,9 \\
\hline
\end{tabular}

\section{Período de Vivero}

Durante 4 meses las plantas fueron regadas con una solución Johnson ("J": $6 \mathrm{ml} / \mathrm{L}$ de $\mathrm{NO}_{3} \mathrm{~K} 1 \mathrm{M}, 4 \mathrm{ml} / \mathrm{L}$ de $\left(\mathrm{NO}_{3}\right)_{2} \mathrm{Ca} 1 \mathrm{M}, 2 \mathrm{ml} / \mathrm{L}$ de $\mathrm{PO}_{4} \mathrm{H}_{2} \mathrm{NH}_{4} 1 \mathrm{M}, 1 \mathrm{ml} / \mathrm{L}$ de $\mathrm{SO}_{4} \mathrm{Mg} .7 \mathrm{H}_{2} \mathrm{O}, 1 \mathrm{ml} / \mathrm{L}$ de micronutrientes, $1 \mathrm{ml} / \mathrm{L}$ Fe-EDTA.

En la primera etapa ( $1^{\circ}-5^{\circ}$ semanas), todas las plantas recibieron la solución "J".

En la segunda etapa $\left(6^{\circ}-10^{\circ}\right.$ semanas), todas las plantas recibieron la solución "J" enriquecida con $\mathrm{P}\left(2 \mathrm{ml} / \mathrm{L} \mathrm{PO}_{4} \mathrm{H}_{2} \mathrm{Na} 1 \mathrm{M}\right)$.

En una tercera etapa $\left(11^{\circ}-14^{\circ}\right.$ semanas), el $50 \%$ de las plantas recibió la solución "J" (K0), mientras que el resto recibió la solución "J" enriquecida con $\mathrm{K}(12 \mathrm{ml} / \mathrm{I} \mathrm{KCl} 1 \mathrm{M})$ (K1).

En la cuarta etapa $\left(15^{\circ}-19^{\circ}\right.$ semanas), la mitad de las plantas recibió riego diario (Cv), mientras que el resto fue regado cada tres días (Ev). En esta etapa, todas las plantas recibieron la solución "J".

Al término de este período, en ocho plantas por tratamiento, se evaluó la biomasa. Se separaron los tallos, las hojas y las raíces y fueron secadas en estufa a $85^{\circ} \mathrm{C}$ por $48 \mathrm{~h}$.

Se determinaron la relación de peso de raíces ( $\mathrm{g}$ raíces/g total, RPR), la relación de peso de hojas ( $\mathrm{g}$ hojas/g total, RPF) y la relación de área foliar ( $\mathrm{cm}^{2}$ hojas/g total, RAF).

A la finalización del período de vivero se estimó variables hídricas utilizando curvas presión-volumen con el método de presurización repetida (Hinckley et al., 1980).

Se utilizó una versión mejorada del programa de Schulte (Schulte y Hinckley, 1985) para 
estimar el potencial osmótico a turgencia plena (חтP) y el módulo de elasticidad de la pared celular $\left(\varepsilon_{\max }\right)$.

El ajuste osmótico ( $\left.\pi_{\mathrm{adj}}\right)$ se calculó como la diferencia entre el $\Pi_{T P}$ del control de cada tratamiento y las otras combinaciones de tratamientos. El ajuste elástico $\left(\varepsilon_{\text {adj }}\right)$ se calculó de manera similar usando $\varepsilon_{\max }$.

\section{Período Plantación}

En la quinta etapa $\left(20^{\circ}-24^{\circ}\right.$ semanas), los plantines de vivero fueron trasplantados a contenedores de mayor tamaño (80 cm altura, $60 \mathrm{~cm}$ diámetro; $200 \mathrm{~L}$ ), con una mezcla de suelo y arena 3:1 (v/v) como sustrato.

Se plantó 11 repeticiones por cada tratamiento de vivero. La mitad de esas plantas fue regada a capacidad de campo diariamente $(\mathrm{Cp})$, y el resto tuvo niveles crecientes de sequía, ya que partiendo de suelo a saturación no se regaron más (Ep).

La biomasa inicial y final se determinó en ocho plantas por tratamiento. La relación de peso de raíces (g raíces/g total, RPR), la relación de peso de hojas ( $\mathrm{g}$ hojas/g total, RPF), la relación de área foliar ( $\mathrm{cm}^{2}$ hojas/g total, RAF) y el área foliar específica $\left(\mathrm{cm}^{2}\right.$ hoja/g.hoja, AFE) y la tasa de asimilación neta $\left(\mathrm{g} / \mathrm{cm}^{2}\right.$ hoja. $t$, TAN) fueron determinadas al final del período de estudio.

Se calculó la tasa de crecimiento relativo (TCR) en biomasa (Hunt, 1982).

Durante el período de plantación de determinó la conductancia estomática $(\mathrm{g})$ al mediodía en cinco plantas por tratamiento utilizando un porómetro (Li 1600, Licor Inc. Lincoln, Ne).

A la finalización del período de plantación se estimaron variables hídricas siguiendo los procedimientos planteados precedentemente.

Para comparar las respuestas de las especies, es decir determinar el grado de cambio de las características medidas en diferentes unidades, se calculó un índice de Plasticidad para algunas variables fisiológicas y morfológicas.

Este índice, que varía entre 0 y 1 se calcula a partir de la diferencia entre el valor máximo medio y mínimo medio que asume cada variable sobre el valor máximo (Valladares et al., 2002).

Se realizó un análisis de variancia multifactorial (niveles de fertilización con $\mathrm{K}$, régimen de riego en vivero y régimen de riego en plantación).

La separación de medias se realizó usando el test de Tukey $(p<0.05)$. Se utilizó el programa Statgraphics versión 6.0 (STSC, Inc. USA 1992).

\section{RESULTADOS}

\section{Período de Vivero}

\section{-Variables Morfológicas}

No se observó efecto del K. Se encontró diferencias significativas en las especies de eucalipto con los niveles de riego (Cuadro $\mathrm{N}^{\circ} 2$ ).

La restricción hídrica no afectó la biomasa ni la alocación relativa en los plantines de $E$. globulus, mientras que la disminuyó significativamente en E. camaldulensis, si bien en esta especie se incrementó la alocación relativa a raíces (Cuadro N 3). 
Cuadro $\mathrm{N}^{\circ} 2$

BIOMASA DE PLANTINES A LA FINALIZACIÓN DE LA ETAPA DE VIVERO

\begin{tabular}{|c|c|c|c|c|c|}
\hline Especie & $\begin{array}{c}\text { Hojas } \\
(\mathrm{g})\end{array}$ & $\begin{array}{c}\text { Tallo } \\
(\mathrm{g})\end{array}$ & $\begin{array}{c}\text { Raíces } \\
(\mathrm{g})\end{array}$ & $\begin{array}{c}\text { Total } \\
(\mathrm{g})\end{array}$ & Tallo - Raíz \\
\hline E. cam - Cv & $4,39 \pm 0,20 a$ & $7,30 \pm 0,47 a$ & $3,04 \pm 0,22 a$ & $14,76 \pm 0,77 a$ & $4,00 \pm 0.20 a$ \\
\hline E. cam - Ev & $3,14 \pm 0,20 b$ & $5,46 \pm 0,48 b$ & $2,60 \pm 0,22 b$ & $11,21 \pm 0,77 b$ & $3,43 \pm 0.19 b$ \\
\hline E. glo - Cv & $3,78 \pm 0,24$ & $3,84 \pm 0,31$ & $1,91 \pm 0,17$ & $9,54 \pm 0,65$ & $4,29 \pm 0.32$ \\
\hline E. glo - Ev & $3,24 \pm 0,24$ & $3,21 \pm 0,31$ & $1,51 \pm 0,17$ & $7,97 \pm 0,65$ & $4,36 \pm 0.32$ \\
\hline $\begin{array}{l}\text { E.cam: } \\
\text { E.glo: } \\
\text { Cv y Ev: }\end{array}$ & $\begin{array}{l}\text { E. camaldulen } \\
\text { E. globulus } \\
\text { Control y restr }\end{array}$ & de riego & vero & & \\
\hline
\end{tabular}

\section{Cuadro $\mathrm{N}^{\circ} 3$}

ALOCACIÓN RELATIVA A HOJAS (RAF, RPF) Y A RAÍCES (RPR)

A LA FINALIZACIÓN DEL PERÍODO DE VIVERO

\begin{tabular}{|c|c|c|c|}
\hline Especie & $\begin{array}{c}\text { RAF } \\
\left(\mathrm{cm}^{2} / \mathrm{g}\right) \\
\end{array}$ & $\begin{array}{c}\text { RPF } \\
\text { (g Hoja/g) } \\
\end{array}$ & $\begin{array}{c}\text { RPR } \\
\text { (g Raíz/g) } \\
\end{array}$ \\
\hline E. cam - Cv & $39,61 \pm 1,85$ & $0,30 \pm 0,01$ & $0,203 \pm 0,006 b$ \\
\hline E. cam - Ev & $36,63 \pm 1,85$ & $0,28 \pm 0,01$ & $0,230 \pm 0,006 a$ \\
\hline E. glo - Cv & $77,42 \pm 3,12$ & $0,40 \pm 0,01$ & $0,197 \pm 0,009$ \\
\hline E. glo - Ev & $78.28 \pm 3,12$ & $0,40 \pm 0,01$ & $0,190 \pm 0,009$ \\
\hline $\begin{array}{l}\text { E.cam: } \\
\text { E.glo: } \\
\text { Cv y Ev: }\end{array}$ & $\begin{array}{l}\text { naldulensis } \\
\text { oulus } \\
\text { y restricción }\end{array}$ & go en vivero & \\
\hline
\end{tabular}

\section{-Variables Fisiológicas}

El $\mathrm{K}$ no modificó las variables fisiológicas analizadas. La restricción hídrica produjo una disminución significativa en el $\Pi_{T P}$ en ambas especies.

$\left.N^{\circ} 4\right)$.

El ajuste osmótico fue 0,20 MPa en E. camaldulensis y 0,19 MPa en E. globulus (Cuadro

Mientras $\varepsilon_{\max }$ no fue modificado en los plantines de $E$. globulus, en los de $E$. camaldulensis se observó un endurecimiento de la pared celular con un ajuste elástico de $4,0 \mathrm{MPa}$ (Cuadro $\mathrm{N}^{\circ} 4$ ).

\section{Cuadro $\mathrm{N}^{\circ} 4$}

POTENCIAL OSMÓTICO A TURGENCIA PLENA $\left(\boldsymbol{n}_{\mathrm{TP}}\right)$ Y MÓDULO DE ELASTICIDAD (EMAX) EN PLANTINES DE EUCALIPTO CON DOS NIVELES DE RIEGO EN VIVERO

\begin{tabular}{|l|c|c|}
\hline Especie & $\begin{array}{c}\pi_{\mathrm{TP}} \\
(\mathrm{MPa})\end{array}$ & $\begin{array}{c}\xi \mathrm{max} \\
(\mathrm{MPa})\end{array}$ \\
\hline
\end{tabular}

\begin{tabular}{|l|l|l|}
\hline E. cam - Cv & $-1,58 \pm 0,07 \mathrm{~b}$ & $17,28 \pm 1,43 \mathrm{~b}$ \\
\hline E. cam - Ev & $-1,78 \pm 0,04 \mathrm{a}$ & $21,33 \pm 1,43 \mathrm{a}$ \\
\hline
\end{tabular}

\begin{tabular}{ll|l|}
\hline E. glo - Cv & $-1,19 \pm 0,08 \mathrm{~b}$ & $13,06 \pm 1,50$ \\
\hline E. glo - Ev & $-1,38 \pm 0,08 \mathrm{a}$ & $12,60 \pm 1,50$ \\
\hline E.cam: & E. camaldulensis \\
E.glo: & E. globulus \\
Cv y Ev: & Control y restricción de riego en vivero
\end{tabular}




\section{Período Plantación}

\section{-Variables Morfológicas}

La restricción de riego impuesta durante la plantación disminuyó significativamente la biomasa de hojas, tallos y la relación T/R en E. camaldulensis (Cuadro $N^{\circ} 5$ ).

No se observó modificaciones en la biomasa de los plantines de E. globulus derivadas de los tratamientos aplicados en el vivero. Solo se encontró efectos producidos por la restricción hídrica durante la plantación con una disminución significativa en la biomasa de hojas y tallos. biomasa foliar.

En cambio, los plantines de E. camaldulensis del nivel $\mathrm{K} 1$ en vivero mostraron mayor

\section{Cuadro $\mathrm{N}^{\circ} 5$ \\ BIOMASA DE PLANTINES DE EUCALIPTO \\ AL FINAL DEL PERÍODO DE PLANTACIÓN}

\begin{tabular}{|l|c|c|c|c|}
\hline Especie & $\begin{array}{c}\text { Hojas } \\
(\mathbf{g})\end{array}$ & $\begin{array}{c}\text { Tallo } \\
(\mathbf{g})\end{array}$ & $\begin{array}{c}\text { Raíces } \\
(\mathbf{g})\end{array}$ & Tallos - Raíz \\
\hline
\end{tabular}

\begin{tabular}{|c|c|c|c|c|}
\hline $\begin{array}{l}\text { E. cam - K0 } \\
\text { E. cam - K1 }\end{array}$ & $\begin{array}{l}7,39 \pm 0,6 b \\
9,18 \pm 0,6 a\end{array}$ & & & \\
\hline E. cam - Cp & $10,3 \pm 0,6 a$ & $17,4 \pm 1,33 \mathrm{a}$ & $8,00 \pm 0,62$ & $3,59 \pm 0.18 a$ \\
\hline E. cam - Ep & $6,2 \pm 0,6 b$ & $12,1 \pm 1,30 b$ & $7,85 \pm 0,62$ & $2,37 \pm 0,18 b$ \\
\hline
\end{tabular}

\begin{tabular}{|l|r|r|r|r|}
\hline E. glo - Cp & $16,03 \pm 1,27 \mathrm{a}$ & $12,42 \pm 0,96 \mathrm{a}$ & $4,74 \pm 0,37$ & $2,59 \pm 0,15$ \\
E. glo - Ep & $9,94 \pm 1,27 \mathrm{~b}$ & $7,80 \pm 0,96 \mathrm{~b}$ & $3,93 \pm 0,38$ & $2,28 \pm 0,15$ \\
\hline
\end{tabular}

E. cam: E. camaldulensis

E. glo: E. globulus

K0 y K1: Niveles de K en vivero

Cp y Ep: Control y restricción de riego en plantación

\section{Tasas de Crecimiento Relativo}

En ambas especies la restricción de riego durante la plantación redujo la tasa de crecimiento en hoja y tallo, pero no modificó la de raíces (Cuadro $\mathrm{N}^{\circ} 6$ ).

Sin embargo, se encontró diferencias en la respuesta de ambas especies de eucalipto como consecuencia de los distintos tratamientos aplicados durante la etapa de vivero. plantas $\mathrm{K} 1$.

Así, en E. globulus solo se observó mayor tasa de crecimiento de hojas en las

Por su parte en E. camaldulensis, las plantas K1 mostraron mayor tasa de crecimiento en biomasa de hojas, tallos y raíces. La restricción hídrica en vivero produjo mayor tasa de crecimiento en hojas y tallo.

\section{Índices de Partición y Eficiencia Foliar}

En esta etapa, no se observó efectos derivados de los tratamientos aplicados en el vivero sobre los índices de alocación relativa en las plantas de E. globulus (Cuadro $\left.\mathrm{N}^{\circ} 7\right)$.

En E. camaldulensis las plantas K1 mostraron mayor TAN, RAF y RPF, mientras que las Ev resultaron con mayor TAN, RAF y AFE.

La restricción hídrica durante la plantación mostró diferente respuesta entre las 
especies. En E. globulus produjo menor RPF y TAN, pero incrementó el área foliar específica (AFE) y la alocación a raíz (RPR). En E. camaldulensis disminuyó el RAF, RPF y la TAN, pero se incrementó el RPR.

\section{Cuadro $\mathrm{N}^{\circ} 6$}

TASA DE CRECIMIENTO RELATIVO EN BIOMASA DE HOJA, TALLO Y RAÍZ EN PLANTINES DE EUCALIPTO A LA FINALIZACIÓN DEL PERÍODO DE PLANTACIÓN

\begin{tabular}{|c|c|c|c|}
\hline Especie & $\begin{array}{c}\text { Hojas } \\
\left(g \cdot g^{-1} \cdot d^{-1}\right)\end{array}$ & $\begin{array}{c}\text { Tallo } \\
\left(g \cdot g^{-1} \cdot d^{-1}\right)\end{array}$ & $\begin{array}{l}\text { Raíces } \\
\left(g \cdot g^{-1} \cdot d^{-1}\right)\end{array}$ \\
\hline E. can & $0,0008 \mathrm{~b}$ & $0,0074 \pm 0,0009 b$ & $0,0093 \pm 0,0008 b$ \\
\hline E. cam - K1 & $0,0096 \pm 0,0008 \mathrm{a}$ & $0,0093 \pm 0,0009 a$ & $0,0122 \pm 0,0008 \mathrm{a}$ \\
\hline E. cam - Cv & $0,0065 \pm 0,0005 b$ & $0,0074 \pm 0.0008 b$ & $0,0108 \pm 0,0008$ \\
\hline E. cam - Ev & $0,0091 \pm 0,0005 a$ & $0,0094 \pm 0,0008 a$ & $0,0112 \pm 0,0008$ \\
\hline E. cam - Cp & $0,0104 \pm 0,0008 \mathrm{a}$ & $0,0103 \pm 0,0009 a$ & $0,0108 \pm 0,0007$ \\
\hline E. cam - Ep & $0,0053 \pm 0,0008 \mathrm{~b}$ & $0.0065 \pm 0.0009 b$ & $0,0107 \pm 0,0007$ \\
\hline E. glo - KO & $0,0115 \pm 0,0008 b$ & $0,0098 \pm 0,0009$ & $0,0085 \pm 0,0008$ \\
\hline E. glo - K1 & $0,0139 \pm 0,0008 \mathrm{a}$ & $0,0103 \pm 0,0009$ & $0,0095 \pm 0,0008$ \\
\hline E. glo - Cv & $0,0122 \pm 0,0009$ & $0,0097 \pm 0,0008$ & $0,0079 \pm 0,0008$ \\
\hline E. glo - Ev & $0,0130 \pm 0,0009$ & $0,0105 \pm 0,0008$ & $0,0102 \pm 0,0008$ \\
\hline E. glo- Cp & $0,0147 \pm 0,0009 a$ & $0,0121 \pm 0,0009 a$ & $0,0096 \pm 0,0007$ \\
\hline E. glo - Ep & $0,0107 \pm 0,0009 \mathrm{~b}$ & $0,0081 \pm 0,0009 b$ & $0,0084 \pm 0,0007$ \\
\hline $\begin{array}{l}\text { E. cam: } \\
\text { E. glo: } \\
\text { Ko y K1: } \\
\text { Cv y Ev: } \\
\text { Cp y Ep: }\end{array}$ & \multicolumn{3}{|c|}{$\begin{array}{l}\text { E. camaldulensis } \\
\text { E. globulus } \\
\text { Niveles de K en vivero } \\
\text { Control y restricción de riego en vivero } \\
\text { Control y restricción de riego en plantad }\end{array}$} \\
\hline
\end{tabular}

Cuadro $\mathrm{N}^{\circ} 7$

ALOCACIÓN RELATIVA A HOJAS (RAF, RPF, AFE), A RAÍCES (RPR) E ÍNDICE DE EFICIENCIA FOLIAR (TAN) EN PLANTINES DE EUCALIPTO A LA FINALIZACIÓN DEL PERÍODO DE PLANTACIÓN

\begin{tabular}{|c|c|c|c|c|c|}
\hline Especie & $\begin{array}{c}\text { TAN } \\
\left(\mathrm{g} / \mathrm{cm}^{2} \text { hoja. } \mathrm{t}\right) \\
\end{array}$ & $\begin{array}{c}\text { RPF } \\
\text { (g hoja/g planta) }\end{array}$ & $\begin{array}{c}\text { AFE } \\
\left(\mathrm{cm}^{2} \text { hoja/g hoja) }\right.\end{array}$ & $\begin{array}{c}\text { RAF } \\
\left(\mathrm{cm}^{2} \text { hoja/g planta) }\right.\end{array}$ & $\begin{array}{c}\text { RPR } \\
\text { (g raíz } / g) \\
\end{array}$ \\
\hline \begin{tabular}{|l} 
E. cam - K0 \\
E. cam - K1
\end{tabular} & $\begin{array}{l}0,00028 \pm 0.00003 \mathrm{~b} \\
0,00036 \pm 0,00003 \mathrm{a}\end{array}$ & $\begin{array}{l}0,255 \pm 0,008 b \\
0,273 \pm 0,008 a\end{array}$ & $\begin{array}{l}82,0 \pm 4,1 \\
83,8 \pm 4,1\end{array}$ & $\begin{array}{l}20,72 \pm 1,00 \mathrm{~b} \\
22,82 \pm 1,00 \mathrm{a}\end{array}$ & $\begin{array}{l}0,270 \pm 0,001 \\
0,256 \pm 0,001\end{array}$ \\
\hline $\begin{array}{l}\text { E. cam - Cv } \\
\text { E. cam - Ev }\end{array}$ & $\begin{array}{l}0,00028 \pm 0,00001 \mathrm{~b} \\
0,00035 \pm 0,00001 \mathrm{a}\end{array}$ & $\begin{array}{l}0,265 \pm 0,007 \\
0,265 \pm 0,007\end{array}$ & $\begin{array}{l}78,1 \pm 4,2 \mathrm{~b} \\
87,6 \pm 4,2 \mathrm{a} \\
\end{array}$ & $\begin{array}{l}20,62 \pm 1,01 \mathrm{~b} \\
22,93 \pm 1,01 \mathrm{a}\end{array}$ & $\begin{array}{l}0,261 \pm 0,001 \\
0,265 \pm 0,001\end{array}$ \\
\hline $\begin{array}{l}\text { E. cam - Cp } \\
\text { E. cam - Ep }\end{array}$ & $\begin{array}{l}0,00036 \pm 0,00001 \mathrm{a} \\
0,00027 \pm 0,00001 \mathrm{~b}\end{array}$ & $\begin{array}{l}0,289 \pm 0,008 \mathrm{a} \\
0,240 \pm 0,008 \mathrm{~b}\end{array}$ & $\begin{array}{l}83,1 \pm 4,2 \\
82,6 \pm 4,2\end{array}$ & $\begin{array}{l}23,90 \pm 1,00 a \\
19,66 \pm 1,00 b\end{array}$ & $\begin{array}{l}0,224 \pm 0,002 b \\
0,301 \pm 0,002 a\end{array}$ \\
\hline $\begin{array}{l}\text { E. glo - K0 } \\
\text { E. glo - K1 }\end{array}$ & $\begin{array}{l}0,00017 \pm 0,00005 \\
0,00020 \pm 0,00005\end{array}$ & $\begin{array}{l}0,473 \pm 0,005 \\
0,486 \pm 0,005\end{array}$ & $\begin{array}{r}128,3 \pm 6,9 \\
135,3 \pm 6,9 \\
\end{array}$ & $\begin{array}{l}60,11 \pm 3,3 \\
65,59 \pm 3,3 \\
\end{array}$ & $\begin{array}{l}0,165 \pm 0,001 \\
0,166 \pm 0,001\end{array}$ \\
\hline $\begin{array}{l}\text { E. glo- Cv } \\
\text { E. glo- Ev }\end{array}$ & $\begin{array}{l}0,00017 \pm 0,00003 \\
0,00019 \pm 0,00003\end{array}$ & $\begin{array}{l}0,478 \pm 0,006 \\
0,482 \pm 0,005 \\
\end{array}$ & $\begin{array}{r}127,8 \pm 6,2 \\
135,7 \pm 6,2 \\
\end{array}$ & $\begin{array}{l}60,91 \pm 3,1 \\
64,79 \pm 3,1 \\
\end{array}$ & $\begin{array}{l}0,158 \pm 0,001 \\
0,160 \pm 0,001\end{array}$ \\
\hline $\begin{array}{l}\text { E. glo - Cp } \\
\text { E. glo - Ep }\end{array}$ & $\begin{array}{l}0,00022 \pm 0,00001 \mathrm{a} \\
0,00014 \pm 0,00001 \mathrm{~b}\end{array}$ & $\begin{array}{l}0,498 \pm 0,008 \mathrm{a} \\
0,462 \pm 0,008 \mathrm{~b}\end{array}$ & $\begin{array}{l}121,4 \pm 5,8 \mathrm{~b} \\
142,2 \pm 5,8 \mathrm{a} \\
\end{array}$ & $\begin{array}{l}59,90 \pm 3,1 \\
64,70 \pm 3,1 \\
\end{array}$ & $\begin{array}{l}0,152 \pm 0,001 \mathrm{~b} \\
0,178 \pm 0,001 \mathrm{a}\end{array}$ \\
\hline
\end{tabular}

$\begin{array}{llll}\text { E. cam: } & \text { E. camaldulensis } & \text { E. glo: } & \text { E. globulus } \\ \text { K0 y K1: } & \text { Niveles de K en vivero } & \text { Cv y Ev: } & \text { Control y restricción de riego en vivero } \\ \text { Cp y Ep: } & \text { Control y restricción de riego en plantación } & \end{array}$

\section{-Variables Fisiológicas}

No se observó efectos derivados de los tratamientos aplicados en el vivero. La restricción hídrica durante la plantación no modificó la gs en las plantas de E. camaldulensis (Cuadro $\mathrm{N}^{\circ} 8$ ), pero la gs de las plantas de $E$. globulus disminuyó significativamente. 


\section{Cuadro $\mathrm{N}^{\circ} 8$}

\section{CONDUCTANCIA ESTOMÁTICA EN PLANTINES DE EUCALIPTO DURANTE EL PERÍODO DE PLANTACIÓN}

\begin{tabular}{|c|c|c|c|}
\hline \multirow[t]{2}{*}{ Especie } & \multicolumn{3}{|c|}{$\begin{array}{l}\text { Conductancia Estomática } \\
\qquad\left(\mathrm{mmol} / \mathrm{m}^{2} . \mathrm{s}\right)\end{array}$} \\
\hline & $15 \mathrm{dpt}$ & $22 \mathrm{dpt}$ & $35 \mathrm{dpt}$ \\
\hline E. cam - Cp & $548,0 \pm 41,6$ & $580,9 \pm 25,6$ & $645,8 \pm 78,0$ \\
\hline E. cam - Ep & $519,8 \pm 41,6$ & $553,8 \pm 25,6$ & $553,7 \pm 78,0$ \\
\hline E. glo - Cp & $577,4 \pm 33,1 \mathrm{a}$ & $699,0 \pm 38,1 \mathrm{a}$ & $704,1 \pm 78,9 a$ \\
\hline E. glo - Ep & $478,2 \pm 33,1 b$ & $426,9 \pm 38,1 b$ & $298,6 \pm 78,9 b$ \\
\hline $\begin{array}{l}\text { E.cam: } \\
\text { E.glo: } \\
\text { Cp y Ep: } \\
\text { Dpt: }\end{array}$ & \multicolumn{3}{|c|}{$\begin{array}{l}\text { E. camaldulensis } \\
\text { E. globulus } \\
\text { Control y restricción de riego en plantación } \\
\text { Días post trasplante }\end{array}$} \\
\hline
\end{tabular}

No se observó modificaciones ni en el $\pi_{T P}$ ni en el $\xi \max$ en los plantines de E. globulus (Cuadro $\mathrm{N}^{\circ}$ 9). En E. camaldulensis el $\Pi_{\mathrm{TP}}$ fue menor en las plantas $\mathrm{K} 1$ y en las Ev, pero no fue modificado por la restricción de riego durante la plantación, sin embargo produjo un ajuste elástico de 2.3 MPa.

\section{Cuadro $\mathrm{N}^{\circ} 9$}

POTENCIAL OSMOTICO A TURGENCIA PLENA $\left(\pi_{\mathrm{TP}}\right)$ Y MODULO MÁXIMO DE ELASTICIDAD ( $\left.\xi m a x\right)$ EN PLANTINES CON DOS NIVELES DE RIEGO EN PLANTACIÓN

\begin{tabular}{|l|c|c|}
\hline Especie & $\begin{array}{c}\Pi_{\mathrm{TP}} \\
(\mathrm{MPa})\end{array}$ & $\begin{array}{c}\text { छmax } \\
(\mathrm{MPa})\end{array}$ \\
\hline
\end{tabular}

\begin{tabular}{|c|c|c|}
\hline E. cam - K0 & $-1,25 \pm 0,02 b$ & $16,95 \pm 0,6$ \\
\hline E. cam - K1 & $-1,32 \pm 0,02 a$ & $17,25 \pm 0,96$ \\
\hline E. cam - Cv & $-1,26 \pm 0,01 b$ & $16,94 \pm 0,94$ \\
\hline E. cam - Ev & $-1,31 \pm 0,01 a$ & $17,27 \pm 0,94$ \\
\hline
\end{tabular}

\begin{tabular}{|l|l|l|}
\hline E. cam - Cp & $-1,27 \pm 0.02$ & $15,93 \pm 0,95 \mathrm{~b}$ \\
\hline E. cam - Ep & $-1,30 \pm 0,02$ & $18,27 \pm 0,95 \mathrm{a}$ \\
\hline
\end{tabular}

\begin{tabular}{|l|l|l|}
\hline E. glo $-\mathrm{K} 0$ & $-0,95 \pm 0.03$ & $6,28 \pm 0,79$ \\
\hline E. glo $-\mathrm{K} 1$ & $-0,92 \pm 0.03$ & $7,15 \pm 0,79$ \\
\hline
\end{tabular}

\begin{tabular}{|l|l|l|}
\hline E. glo - Cv & $-0,93 \pm 0,02$ & $6,94 \pm 0,77$ \\
\hline E. glo - Ev & $-0,93 \pm 0,02$ & $6,80 \pm 0,77$ \\
\hline
\end{tabular}

\begin{tabular}{|l|l|l|}
\hline E. glo - Cp & $-0,91 \pm 0,03$ & $6,54 \pm 0,79$ \\
\hline E. glo - Ep & $-0,95 \pm 0,03$ & $7,20 \pm 0,79$ \\
\hline E.cam: & E. camaldulensis \\
E.glo: & E. globulus \\
Cv y Ev: & Control y restricción de riego en vivero \\
Cp y Ep: & Control y restricción de riego en plantación.
\end{tabular}

\section{-Índices de Plasticidad}

El potasio y la disponibilidad hídrica durante la etapa de vivero produjeron modificaciones plásticas de distinto nivel, tanto morfológicas como fisiológicas, en los plantines de ambas especies de eucalipto. La disponibilidad hídrica presentó los mayores valores en el índice de plasticidad en todas las variables evaluadas durante la etapa de vivero (Cuadro $\mathrm{N}^{\circ} 10$ ). En la etapa postplantación, los mayores índices correspondieron al efecto de la restricción de riego durante la 
plantación, pero también se observó diferencias entre las especies de eucalipto apreciándose un mayor efecto del nivel de $\mathrm{K}$ en E. camaldulensis (Cuadro $\mathrm{N}^{\circ} 10$ ).

\section{Cuadro $\mathrm{N}^{\circ} 10$ \\ INDICES DE PLASTICIDAD FENOTÍPICA PARA VARIABLES RESPUESTA FISIOLÓGICAS Y MORFOLÓGICAS EN PLANTINES DE EUCALIPTO. DISTINTOS NIVELES DE FERTILIZACIÓN CON K Y DISPONIBILIDAD HÍDRICA EN LA ETAPA DE VIVERO Y EN LA DE PLANTACIÓN}

\begin{tabular}{|l|c|c|c|c|}
\hline \multirow{2}{*}{ VIVERO } & \multicolumn{2}{|c|}{ E. camaldulensis } & \multicolumn{2}{c|}{ E. globulus } \\
\cline { 2 - 5 } & Potasio & Riego & Potasio & Riego \\
\hline
\end{tabular}

\begin{tabular}{|c|c|c|c|c|}
\hline Fisiológicas & \multicolumn{5}{l|}{} \\
\hline Pot. osmótico & 0,005 & 0,112 & 0,053 & 0,137 \\
\hline Mód. elasticidad & 0,021 & 0,189 & 0,105 & 0,034 \\
\hline Promedio & 0,013 & 0,150 & 0,079 & 0,085 \\
\hline
\end{tabular}

\begin{tabular}{|l|l|l|l|l|}
\hline Morfológicas & \multicolumn{5}{|l|}{} \\
\hline Biomasa hoja & 0,100 & 0,284 & 0,152 & 0,142 \\
\hline Biomasa de tallo & 0,045 & 0,252 & 0,134 & 0,164 \\
\hline Biomasa de raíces & 0,168 & 0,144 & 0,122 & 0,157 \\
\hline Promedio & 0,104 & 0,220 & 0,136 & 0,154 \\
\hline
\end{tabular}

\begin{tabular}{|l|c|c|c|c|c|c|}
\hline \multirow{2}{*}{ PLANTACION } & \multicolumn{3}{|c|}{ E. camaldulensis } & \multicolumn{3}{c|}{ E. globulus } \\
\cline { 2 - 7 } & Potasio & $\begin{array}{c}\text { Riego } \\
\text { Vivero }\end{array}$ & $\begin{array}{c}\text { Riego } \\
\text { Plantación }\end{array}$ & Potasio & $\begin{array}{c}\text { Riego } \\
\text { Vivero }\end{array}$ & $\begin{array}{c}\text { Riego } \\
\text { Plantación }\end{array}$ \\
\hline
\end{tabular}

\begin{tabular}{|c|c|c|c|c|c|c|}
\hline \multicolumn{1}{|l|}{ Fisiológicas } & & \\
\cline { 1 - 5 } Pot. osmótico & 0,030 & 0,015 & 0,045 & 0,030 & 0,006 & 0,046 \\
\hline Mód. elasticidad & 0,017 & 0,018 & 0,128 & 0,012 & 0,020 & 0,090 \\
\hline Promedio & 0,023 & 0,016 & 0,086 & 0,076 & 0,013 & 0,060 \\
\hline
\end{tabular}

\begin{tabular}{|l|l|l|l|l|l|l|}
\hline \multicolumn{1}{|l|}{ Morfológicas } & 0,195 & 0,070 & 0,391 & 0,028 & 0,063 & 0,379 \\
\hline Biomasa hoja & 0,135 & 0,070 & 0,303 & 0,002 & 0,103 & 0,371 \\
\hline Biomasa de tallo & 0,081 & 0,062 & 0,018 & 0,006 & 0,022 & 0,170 \\
\hline Biomasa de raíces & 0,137 & 0,046 & 0,237 & 0,012 & 0,062 & 0,306 \\
\hline Promedio
\end{tabular}

\section{DISCUSION Y CONCLUSIONES}

La restricción hídrica durante la etapa de plantación produjo una disminución significativa en la biomasa de ambas especies. La reducción en la acumulación de biomasa (especialmente de hojas y tallos) y en el crecimiento en plantas de eucalipto sometidas a restricción de riego es una respuesta conocida (Osorio et al., 1998; Lemcoff et al., 2002; Guarnaschelli et al., 2003; Guarnaschelli et al., 2006).

Sin embargo, las prácticas de aclimatación aplicadas en la etapa de vivero, tendientes a disminuir ese efecto, mostraron resultados diferentes entre E. camaldulensis y E. globulus. Al término de esa etapa la restricción hídrica no afectó la biomasa en los plantines de E. globulus, y si bien disminuyó la biomasa de los tallos, las hojas y las raíces en las plantas de E. camaldulensis, en las plantas Ev de dicha especie se observó una mayor alocación relativa hacia las raíces, siendo este tipo de respuesta similar a la observada por Pereira y Chaves (1993).

También se observó respuestas de aclimatación fisiológica. En concordancia con resultados de otros autores (Tuomela, 1997; Guarnaschelli et al., 2001; Lemcoff et al., 2002; Merchant et al., 2007) se observó ajuste osmótico en ambas especies de eucalipto y, además, un endurecimiento de la pared celular en E. camaldulensis. La capacidad de realizar ajuste osmótico puede considerarse una respuesta de aclimatación a la sequía (Geiger y Servaites, 1991) permitiendo mantener la presión de turgencia para mantener los estomas abiertos y admitir el 
ingreso de dióxido de carbono para la fotosíntesis (Nilsen y Orcutt, 1996). Un mayor módulo de elasticidad estabiliza el contenido hídrico y ayuda a mantener la diferencia entre los potenciales agua del suelo y de la planta favoreciendo la absorción de agua sin cambios significativos en el contenido hídrico de los tejidos (Nilsen y Orcut, 1996). En este trabajo las plantas de E. globulus solo mostraron una aclimatación fisiológica derivada de la realización de ajuste osmótico en contraste con trabajos previos (Guarnaschelli et al., 2003; Guarnaschelli et al., 2006). No siempre se observa diferencias en las variables hídricas en plantas de $E$. globulus regadas y no regadas, y respuestas como el ajuste osmótico y/o elástico no son consistentes en esta especie (White et al., 1996), dependiendo de la subespecie (Wang et al., 1988), de la época del año (Correia et al., 1989) o el clon analizado (Osorio et al., 1998). En este ensayo, se produjo ajuste osmótico y este resultado es contrario al encontrado por Wang et al. (1988) con la misma procedencia (Flinders Island). Correia et al. (1989) tampoco observaron efecto de la fertilización en las variables hídricas ante una situación de sequía en plantas de E. globulus.

En ningún caso se observó, en esta etapa, efecto de la mayor dosis de K. En relación a lo anterior, los mayores índices de plasticidad como respuesta a la restricción hídrica con relación a los derivados del agregado de $\mathrm{K}$ indican también que el régimen de riego tuvo más impacto sobre los atributos de las plantas evaluados en este estudio. A su vez, y en general, los valores de los índices resultaron mayores en E. camaldulensis que en E. globulus. La plasticidad fenotípica es una característica variable entre especies (Valladares et al., 2005; Castro-Diez et al., 2006; Guarnaschelli et al., 2012), entre poblaciones de una misma especie (Guarnaschelli et al., 2001; Guarnaschelli et al., 2006) y dependiente del gradiente de recursos disponibles para las plantas (Valladares et al., 2002).

En la etapa de plantación continuaron manifestándose diferencias en las respuestas de ambas especies y las prácticas de riego y fertilización aplicadas en la etapa de vivero tuvieron particular influencia en el comportamiento de las plantas de E. camaldulensis después del trasplante. Si bien la menor disponibilidad de agua durante la plantación disminuyó la biomasa aérea en esta especie, las mayores tasas de crecimiento aéreo y radical se observaron en plantines que habían sido sometidos a un período de restricción hídrica en vivero y que recibieron una mayor dosis de $\mathrm{K}$. Los efectos positivos del agregado de $\mathrm{K}$ sobre respuestas morfológicas y fisiológicas en distintas especies fueron presentados por varios autores. En plantas de E. globulus el agregado de $\mathrm{K}$ produjo un descenso adicional en el potencial osmótico a turgencia plena (Guarnaschelli et al., 2010 y 2012). En otros trabajos también se destaca el efecto del K mejorando la tolerancia a la sequía, la eficiencia en el uso del agua, en el crecimiento y la aclimatación de las plantas (Eakes et al., 1991; Egilla et al., 2005).

Dentro del sistema de producción forestal es muy importante que las plantas tengan una buena respuesta al trasplante, y en particular ante condiciones de baja disponibilidad hídrica, manifestada a través de sus tasas de crecimiento. En general, las plantas más pequeñas tienen TCR altas que luego tienden a disminuir. Por lo tanto, los plantines que fueron sometidos a restricción hídrica durante la etapa de vivero pueden tener mayores TCR simplemente debido a su menor tamaño al momento de trasplante.

Sin embargo una suma de respuestas fisiológicas y morfológicas podría explicar estas mayores tasas de crecimiento observadas en las plantas K1 y Ev de E. camaldulensis a partir de mejorar la disponibilidad de agua para las mismas; ajuste osmótico, el endurecimiento de las paredes celulares, una mayor proporción de biomasa destinada a raíces y mantenimiento de la conductancia estomática durante la etapa de restricción hídrica en la plantación.

Por otro lado, la tasa de crecimiento relativo también puede explicarse en función de un mayor rendimiento fotosintético (mayor TAN), mayor distribución relativa de fotosintatos hacia el área foliar (mayor RAF), o por combinación de ambas. En tal sentido, las plantas de $E$. camaldulensis $\mathrm{K} 1$ y Ev mostraron mayor TAN y mayor RAF.

En las condiciones de restricción hídrica durante la plantación, el área foliar específica no se modificó en E. camaldulensis y aumentó significativamente en E. globulus. En especies leñosas (entre ellas Eucalyptus) el área foliar específica muestra respuestas contrastantes ante condiciones de estrés hídrico. Puede disminuir (Pita y Pardos, 2001; Ngugi et al., 2003) o bien aumentar 
(Galmes et al., 2005). El estrés hídrico disminuyó la alocación relativa a hojas en ambas especies de manera similar a lo encontrado por Gindabaa et al. (2005), pero en contraste con esos autores se encontró un aumento en la alocación relativa a raíz (cabe destacar que ya se había producido un aumento similar en $E$. camaldulensis en la etapa de vivero).

Tanto en la finalización de la etapa de vivero como en la de plantación los índices de plasticidad morfológica resultaron superiores a los fisiológicos en ambas especies. Galmes et al. (2005) postulan que los ajustes morfológicos se producirían solo en los estados tempranos de desarrollo de las plantas leñosas. Sin embargo, en otros trabajos, y en distintas especies, se reportan mayores índices de plasticidad fisiológica (Valladares et al., 2000; Guarnaschelli et al., 2010).

Para las condiciones de este ensayo y de acuerdo a los valores hallados para los índices de plasticidad, E. camaldulensis mostró mayores valores de ajuste morfológico que E. globulus. Estos resultados resultan contradictorios si se considera que en general se acepta que la plasticidad es mayor en plantas con ambientes más ricos en recursos (Grime et al., 1986). En términos de precipitaciones la semilla de E. camaldulensis utilizada es de una zona con $452 \mathrm{~mm}$ anuales y la de E. globulus de una zona con 743. Sin embargo, Aikio y Markkola (2002) establecen lo contrario y para estos autores las plantas son más plásticas si proviene de ambientes con menos recursos. En ambientes ricos las plantas no necesitarían plasticidad para modificar su alocación relativa; con recursos limitados un cambio de alocación en concordancia con el recurso limitante puede conducir a incrementos en las tasas de crecimiento.

El establecimiento de los plantines forestales es una etapa crítica que depende fuertemente de la disponibilidad de agua. Las plantas trasplantadas sufren un estrés fisiológico severo porque su capacidad de absorción de agua se ve fuertemente disminuida (Kozlowski y Pallardy, 2002). Es fundamental que las plantas manifiesten una buena respuesta al trasplante a través de sus tasas de crecimiento.

La información presentada sugiere que prácticas de manejo diferencial de recursos durante la etapa de vivero pueden generar respuestas de aclimatación con efectos beneficiosos post-trasplante. Así, períodos de baja disponibilidad de agua y una mayor disponibilidad de $\mathrm{K}$ mejoran el crecimiento de los plantines de eucalipto después del trasplante. Sin embargo, y considerando que en el caso de $E$. globulus las respuestas fueron distintas e inclusive sin diferencias significativas con los controles la aplicación de dichas prácticas culturales debería ajustarse en función de cada especie.

Los resultados de este trabajo aportan información que permitiría ajustar programas de fertilización y aclimatación a la sequía en vivero y destacan la necesidad de regular y/o adecuar las tareas de acuerdo a la especie en cuestión.

\section{REFERENCIAS}

Aikio, S.; Markkola, A., 2002. Optimality and phenotypic plasticity of shoot-to-root ratio under variable light and nutrient availabilities. Evol. Ecol. 16: 67-76, 2002.

Burdett, A. N., 1990. Physiological processes in plantation establishment and the development of specification for forest planting stock, Can. J. For. Res. 20: 415-427.

Chamshama, S. A. and Hall, J. B., 1987. Effects of nursery treatments on Eucalyptus camaldulensis field establishment and early growth at Mafiga, Morogoro, Tanzania. For. Ecol. Manage. 21: 91-108.

Castro-Dıez, P.; Navarro, J.; Pintado, A.; Sancho, L. G. and Maestro, M., 2006. Interactive effects of shade and irrigation on the performance of seedlings of three Mediterranean Quercus species. Tree Physiol., 26: 389400

Correia, M.; Torres, F. and Pereira, J., 1989. Water and nutrient supply regimes and the water relations of juvenile leaves of Eucalyptus globulus. Tree Physiol. 5: 459-475

Cozzo, D., 1995. Silvicultura de plantaciones maderables. Orientación Gráfica Editora. Buenos Aires, Argentina 
Eakes, D. J.; Wright, R. D. and Seiler, R., 1991. Potassium nutrition and moisture stress tolerance of Salvia. Hort. Science 26: 422,

Egilla, J. N.; Davies, F. T. and Boutton, T. W., 2005. Drought stress influences leaf water content, photosynthesis, and water-use efficiency of Hibiscus Rosa sinensis at three potassium concentrations. Photosynth. 43 (1): 135-140.

Galmes, J.; Cifre, J.; Medrano, H. and Flexas, J., 2005. Modulation of relative growth rate and its components by water stress in Mediterranean species with different growth forms.Oecol. 145: 21-31

Garau, A. M; Guarnaschelli, A. B.; Mema, V.; Pathauer, P. and Lemcoff, J. H., 2004. Tissue water relations in Eucalyptus seedlings: Effects of species, $\mathrm{K}$ fertilization and drought. Conferencia Internacional IUFRO Eucalyptus in a Changing World. Aveiro, Portugal.

Garau, A. M., 2012. Los recursos forestales en el mundo y Argentina. C.I.F.A. Facultad Agronomía. UBA. 16 pgs.

Geiger, D. and Servaites, J., 1991. Carbon allocation and response to stress. Páginas 103-127 en H. Mooney, W. Winner y E. Pell, editores. Response of plants to multiple stresses. Academic Press, New York, USA.

Gindabaa, J.; Rozanovb, A. and Negashc, L., 2005. Photosynthetic gas exchange, growth and biomass allocation of two Eucalyptus and three indigenous tree species of Ethiopia under moisture deficit. For. Ecol. Manage. 205: 127-138

Grime, J. P.; Crick, J. C. and Rincon, J. E., 1986. The ecological significance of plasticity. In D.H. Jennings and A.J. Trewavas (eds) Plasticity in Plants pp. 5-29. Symposia of the Society for Experimental Biology No. 40. Company of Biologists, Cambridge.

Guarnaschelli, A. B.; Lemcoff, J. H.; Prystupa, P. and Basci, S., 2003. Responses to drought preconditioning in Eucalyptus globulus Labill. provenances. Trees 17: 501-509.

Guarnaschelli, A. B.; Prystupa, P. and Lemcoff, J. H., 2006. Drought conditioning improves water status, stomatal conductance and survival of Eucalyptus globulus subsp. bicostata. Ann. For. Sci. 63: 941-950.

Guarnaschelli, A. B.; Garau, A. M. y Caccia, F., 2006. Respuestas al déficit hídrico y lumínico durante el establecimiento de Salix. Jornadas de Salicáceas. Buenos Aires.

Guarnaschelli, A. B.; Ruiz Nuñez, J.; Chiavassa, J. A.; Fedotova, N. y Garau, A. M., 2010. Aclimatación en vivero en plantas de Eucalyptus por restricción hídrica y fertilización potásica. XXIV Jornadas Forestales de Entre Ríos. Concordia, Entre Ríos.

Guarnaschelli, A. B.; Garau, A. M.; Caccia, F. D. and Cortizo, S. C., 2008. Physiological responses to shade and drought in young Willow plants. 23rd Session of the International Poplar Commission. "Poplars, willows and people's wellbeing". Beijing, China.

Guarnaschelli, A. B.; Chiavassa, J. A. y Garau, A. M., 2012. Respuestas fisiologicas, resistencia al estrés y crecimiento de plantas de Eucalyptus globulus y Eucalyptus grandis modificadas por potasio bajo condiciones de sequía. Congreso Latinoamericano de Eucalyptus. Pucón, Chile.

Hunt, R., 1982. Plant growth curves. The functional approach to plant growth analysis. University Park Press, Baltimore, Gran Bretaña.

Kleiner, K. W.; Abrams, M. D. and Schultz, J. C., 1992. The impact of water and nutrient deficiencies on the growth, gas exchange and water relations of Red Oak and Chestnut Oak. Tree Physiol 11:271-287

Kozlowski, T. and Pallardy, S. G., 1997. Physiology of woody plants. Second Edition. Academic Press, Nueva York.

Kozlowski, T. T. and Pallardy, S. G., 2002. Acclimation and adaptive responses of woody plants to environmental stresses. Bot. Rev. 68: 270-334.

Lambers, H. and Poorter, H., 1992. Inherent variation in growth rate between higher plants: A search for ecological causes and consequences. Adv. Ecol. Res. 23:187-261

Landis, T. D., 1989. Irrigation and water management. En Landis, T. D.; Tinus, R. W.; McDonald, S. E. y Barnett, J. P. The Container Tree Nursery Manual, vol. 4. Agric. Handbook. 674. Washington, D. C. USDA Forest Service: $1-39$. 
Lemcoff, J; Guarnaschelli, A; Garau, A.; Basciali, M. and Ghersa, C., 1994. Osmotic adjustment and its use as selection criterion in Eucalyptus seedlings. Can. J. For. Res. 24:2404-2408.

Lemcoff, J; Guarnaschelli, A; Garau, A. and Prystupa, P., 2002. Elastic and osmotic adjustment in rooted cuttings of several clones of Eucalyptus camaldulensis Dehnh. from southeasterm Australia after a drought. Flora. 197:134-142.

Margolis, H. A. and Brand, D. G., 1990. An ecophysiological basis for understanding plantation establishment. Can.J.For.Res. 20: 375-390.

Marschner, H., 1995. Mineral Nutrition of Higher Plants. 2nd Ed. Academic Press, San Diego, California, USA.

Mema, V.; Garau, A.; Guarnaschelli, A. y Lemcoff, J., 2003. Fertilización de Eucalyptus globulus en vivero: Modificaciones morfológicas y fisiológicas inducidas por diferentes niveles de fertilización nitrogenada y potásica y su relación con la tolerancia al estrés hídrico en plantación. Primer Simposio lbearoamericano de Eucalyptus globulus. Montevideo, Uruguay.

Merchant, A.; Callister, A.; Arndt, S.; Tausz, M. and Adams, M., 2007. Contrasting physiological responses to water deficit in six Eucalyptus species. Ann. Bot. 100: 1507-1515.

Nilsen, E. and Orcutt, D., 1996. The physiology of plants under stress. Wiley, Nueva York.

Ngugi, M. R.; Hunt, A.; Doley, D.; Ryan, P. and Dart, P., 2003. Dry matter production and allocation in Eucalyptus cloeziana and Eucalyptus argophloia seedlings in response to soil water déficits. New For. 26: 187200, 2003.

Osório, J.; Osório, M. L.; Chaves, M. and Pereira, J. S., 1998. Effects of water deficits on 13C discrimination and transpiration efficiency of Eucalyptus globulus clones. Aust. J. Plant Physiol.25: 645-653

Pereira, J. S, and Chaves, M. M., 1993. Plant water deficits in Mediterranean ecosystems. In Water Deficits: Plant Responses from Cell to Community. J. A. C. Smith y H. Griffiths, Eds. Bios Scientific Publishers, Oxford. Págs. 237-250

Plante, E.; Garau, A. y Lemcoff, J. H., 2002. Fertilización diferencial con potasio en vivero. Modificaciones morfológicas y fisiológicas en plantines de eucalipto. XI Reunión Latinoam. Fisiología Vegetal- XXIV Reunión Argentina de Fisiología Vegetal- I Congreso Uruguayo de Fisiología Vegetal.

Pita, P. and Pardos, J., 2001. Growth, leaf morphology, water use and tissue water relations of Eucalyptus globulus clones in response to water deficit. Tree Physiol. 21: 599-607.

Reis, G.; Reis, M. e Maestri, M., 1988. Crescimento e relaçoes hidricas de mudas de Eucalyptus grandis e Eucalyptus camaldulensis em tubetes sob tres regimes de irrigaçao. Rev. Arbore 12: 183-195.

Sasse, J. and Sands, R., 1996. Comparative responses of cuttings and seedlings of Eucalyptus globulus to water stress. Tree Physiol. 16: 287-294

Schulte, P. and Hinckley, T., 1985. A comparison of pressure-volume curve data analysis technique. Tree Physiol. 2: 89-103

Soriano, A., 1991. Río de la Plata grasslands. Páginas 367-407 en R. Coupland, editor. Ecosystems of the world. Natural Grasslands. Introduction and Western Hemisphere. Elsevier. Amsterdam.

Teixeira, P. C.; Moraes Gonçalves, J. L.; Arthur Junior, J. C. and Dezordi, C., 2008. Eucalyptus sp. seedling response to potassium fertilization and soil water: Matéria seca e relações hídricas em mudas de Eucalyptus sp. em função da fertilização potássica e da umidade do solo .Ciência Flor., 18 (1): 47-63

Tuomela, K., 1997. Leaf water relations in six provenances of Eucalyptus microtheca: A greenhouse experiment. For. Ecol. Manage. 92 (1-3): 1-10

Valladares, F.; Wright, S. J.; Lasso, E.; Kitajima, K. and Pearcy, W.. 2000. Plastic phenotypic response to light of 16 congeneric shrubs from a Panamanian rainforest. Ecol. 81:1925-1936

Valladares, F.; Chico, J. M.; Aranda, I.; Balaguer, L.; Dizengremel, P.; Manrique, E. and Dreyer, E., 2002. The greater seedling high-light tolerance of Quercus robur over Fagus sylvatica is linked to a greater physiological plasticity. Trees 16: 395-403. 
Valladares, F.; Dobarro, I.; Sanchez-Gomez, D. and Pearcy, R., 2005. Photoinhibition and drought in Mediterranean Woody saplings: scaling effects and interactions in sun and shade phenotypes. Jour. Exper. Bot., 56: $483-494$

van den Driessche, R., 1991. Influence of container nursery regimes on drought resistance of seedlings following planting. I. Survival and growth, Can. J. For. Res. 21: 555-565.

van den Driessche, R., 1992. Changes in drought resistance and root growth capacity of container seedlings in response to nursery drought, nitrogen and potassium treatments, Can. J. For. Res. 22: 740-749.

von Wernich, M. y Lavado, R., 2001. El potasio en viveros forestales de eucaliptos. En Actas del Primer Simposio FAUBA-IPI-Fertilizar INTA. Págs. 199-205.

Wang, D.; Bachelard E. and Banks, J., 1988. Growth and water relations of seedlings of two subspecies of Eucalyptus globulus. Three Physiol. 4:129-138.

White, D.; Beadle, C. and Worledge, D., 1996. Leaf water relations of Eucalyptus globulus ssp. globulus and $E$. nitens: seasonal, drought and species effects. Tree Physiol.16: 469-476.

White, D.; Turner; N. and Galbraith, J., 2000. Leaf water relations and stomatal behavior of four allopatric Eucalyptus species planted in Mediterranean southwestern Australia. Tree Physiol. 20:1157-1165 
